\title{
Wandel und Perspektiven der diagnostischen Onkoradiologie
}

\author{
W. Golder \\ Klinik für Radiologie und Nuklearmedizin, Klinikum Benjamin Franklin, Freie Universität, Berlin
}

Die diagnostische Onkoradiologie hat sich in den letzten 25 Jahren wesentlich verändert. Der Wandel wird an der Fülle des Bildmaterials und der Vielfalt der angewandten Techniken sichtbar. Im Verlauf vieler Tumorleiden werden - einmal oder mehrfach, simultan oder sukzessive - diverse bildgebende Verfahren in Anspruch genommen. Den aus Bildern gewonnenen Informationen kommt eine ähnlich große Bedeutung zu wie den Resultaten der Labortests, der Endoskopie und der feingeweblichen Untersuchungen. Die Tumornachweisverfahren der konventionellen Röntgendiagnostik, auf die sich der Radiologe bis vor einem Vierteljahrhundert gestützt hat, sind heute mit wenigen Ausnahmen nicht mehr in Gebrauch. Nur die biplane Röntgenuntersuchung des Thorax, des Abdomen und des Skeletts hat sich sowohl in akuten Situationen als auch im Rahmen von Verlaufskontrollen für die Beantwortung onkologischer Fragen behaupten können.

Die apparativen und methodischen Fortschritte der Bildgebung haben der Onkoradiologie über die rein diagnostische Arbeit hinaus weitere Tätigkeitsbereiche erschlossen. Zwar bilden die Entdeckung, Stadieneinteilung und Therapiekontrolle maligner Tumoren unverändert das Kernpotenzial der Disziplin; die Differenzialtherapie, insbesondere auch die Entscheidung für einen Wechsel der Behandlungsstrategie, wird durch die radiologische Information vielfach wesentlich beeinflusst. An den Flanken des Faches haben sich aber mit der Früherkennung und der radiologisch gesteuerten minimal invasiven Therapie maligner Tumoren zwei leistungsfähige neue Segmente etablieren können. Die Verfahren der radiologischen Früherkennung sind deshalb so erfolgreich, weil sie nicht nur leistungsfähig und wenig invasiv sind, sondern weil sie gerade die besonders häufigen Organkrebse erfassen. Innerhalb der Methoden der minimal invasiven radiologischen Tumortherapie sind zwei große Gruppen zu unterscheiden. Erstere umfasst jene im Allgemeinen kathetergesteuerten Prozeduren, die die chirurgische Therapie erleichtern bzw. die Wirksamkeit der medikamentösen Therapie verbessern sollen, letztere die Verfahren, die mit operativen Eingriffen in Wettstreit treten. Während die Interventionen der ersten Gruppe nahezu ausschließlich von Radiologen durchgeführt werden, reklamieren den Zugriff auf letztere auch andere klinische Disziplinen.

Der Fortschritt in der diagnostischen Onkoradiologie vollzieht sich unter der Ägide der physikalischen Forschung und der technischen Innovationen. Die elektromedizinische und die pharmazeutische Industrie bestimmen den Takt der Entwicklung. Die Verfügbarkeit neuer Hard- und Software lässt klinische Fragestellungen zugleich entstehen und beantworten. Auf diesem Fundament ruht der wissenschaftliche Studienbetrieb. Durch die bei der Applikation gewonnenen Erfahrungen werden einerseits die initialen Evaluationskonzepte modifiziert, andererseits Änderungs- und Ausbaumöglichkeiten der Geräte sichtbar. Außerdem lernt man die Chancen der Kombination bzw. des gestaffelten Einsatzes der verschiedenen Verfahren kennen. Auf diesem Feld arbeitet die forschende Onkoradiologie mit großem Erfolg.

\section{Von der konventionellen zur digitalen Radiographie}

Der Wandel der diagnostischen Onkoradiologie ist durch den Übergang von der konventionellen zur digitalen Radiographie wesentlich gefördert worden. Da letzterer noch keineswegs abgeschlossen ist, wird die Tumordiagnostik auch weiterhin davon profitieren. Die Digitalisierung der Projektionsbilder und durchleuchtungsgezielten Röntgenaufnahmen spielt dabei allerdings nur eine untergeordnete Rolle. Auch die digitale Subtraktionsangiographie (DSA) ist trotz der überragenden Bedeutung der Methode für die Gefäßdiagnostik aus der Sicht des diagnostischen Onkoradiologen nachgeordnet. Die geringe Wertschätzung ist allerdings nicht der Methode an sich zuzuschreiben, sondern dem Bedeutungsverlust der selektiven Angiographie für die Tumorsuche und -charakterisie-

\begin{tabular}{ll}
\hline KARGER & ( 2002 S. Karger GmbH, Freiburg \\
$\begin{array}{l}\text { Fax +497614520714 } \\
\begin{array}{l}\text { E-mail Information@Karger.de } \\
\text { www.karger.com }\end{array}\end{array}$ & Accessible online at: \\
www.karger.com/journals/onk
\end{tabular}

Prof. Dr. W. Golder

Klinik für Radiologie und Nuklearmedizin

Klinikum Benjamin Franklin, Freie Universität

Hindenburgdamm 30

D-12200 Berlin (Deutschland) 
rung an sich. Die Zeit, in der man z.B. kleine Pankreastumoren angiographisch nachzuweisen versuchte, ist jedenfalls vorbei. Für derartige Aufgaben stehen heute die Schnittbildverfahren zur Verfügung, die allesamt primär digitale Techniken sind.

Die dem Volumen nach bei weitem dominierende und zugleich am wenigsten an die Radiologie als selbständiges Fach gebundene Methode ist die Ultrasonographie (US). Unzählige Untersuchungen belegen ihre Bedeutung für den Nachweis primärer und sekundärer Tumoren und deren Verlaufskontrolle nach bzw. unter Therapie. Es gibt keine Disziplin mehr, die ihre onkologische Klientel nicht wenigstens initial und im weiteren Verlauf mehr oder weniger regelmäßig sonographiert. Nur gelegentlich verlässt man sich freilich ausschließlich auf das Sonogramm. Die Abhängigkeit der Befundsicherheit von den Untersuchungsbedingungen und der Erfahrung des Arztes sowie die gelegentlich mangelhafte Dokumentation der Ultraschallbilder tragen zu dieser Haltung bei. Technische Fortentwicklungen des Verfahrens (Farbkodierung, 3D-Visualisierung, «tissue harmonic imaging») und der Einsatz spezifischer Kontrastmittel haben jedoch die Bildqualität verbessert und neue Anwendungsbereiche eröffnet. Die Position des Ultraschalls in der onkoradiologischen Diagnostik hat sich dadurch gefestigt.

Die für den Tumorradiologen optimale Kombination aus weiter Verbreitung der Geräte, überlegener anatomischer Orientierung, hervorragender räumlicher und Kontrastauflösung und exzellenter Eignung für die Differenzialdiagnostik bietet die Computertomographie (CT). Auch kleine Läsionen, d.h. solche mit weniger als $10 \mathrm{~mm}$ Durchmesser, werden sicher erkannt und können vielfach zuverlässig charakterisiert werden. Für den Onkoradiologen spielt dabei neben der Größen- bzw. Volumenbestimmung die Dichtemessung vor, während und nach Applikation jodhaltiger Kontrastmittel die entscheidende Rolle. Die daher naheliegende Kombination aus CT und Angiographie hat sich allerdings nicht durchgesetzt. Die Spiral-CT beschleunigt den Ablauf der Untersuchung, liefert lückenlose Datensätze und eröffnet Möglichkeiten zur Nachbearbeitung der Querschnittsbilder (multiplanare Rekonstruktion, 3D-Darstellung, so genannte virtuelle Endoskopie). Für die bessere Bildqualität und überlegene diagnostische Aussage muss jedoch gelegentlich eine höhere Strahlenexposition in Kauf genommen werden, ein Preis, der auch bei Tumorkranken kritisch zu bewerten ist. Die jüngste technische Variante ist die so genannte Mehrschicht-Spiral-CT, die das Verfahren weiter beschleunigt und den Einsatz des Kontrastmittels ökonomisiert; ihre klinische Erprobung bei der onkologischen Klientel ist in vollem Gange.

Mit der Magnetresonanztomographie (MRT) hat die Onkoradiologie ein Instrument erhalten, das ebensoviel und noch weitaus mehr leistet als die CT, und dies ohne Exposition des Patienten gegenüber ionisierenden Strahlen, und das dennoch nicht zum alles beherrschenden Verfahren geworden ist. Diese Entwicklung hat sowohl logistische und ökonomische als auch medizinische Gründe. Sie auseinander zu halten fällt vielfach schwer. Am weitesten von der CT abgesetzt hat sich die MRT bei der Erkennung der Tumoren des Zentralnervensystems. In diesem Bereich ist die Dominanz der Methode so eindrucksvoll, dass mögliche organisatorische Probleme bei der praktischen Durchführung bereitwillig in Kauf genommen werden. Eine ähnlich führende Rolle spielt die MRT in der Onkoradiologie des Skeletts. Auch bei der Suche nach Knochenfiliae vertraut man mehr und mehr den MR-Schnittbildern. Dagegen wird die MR-Diagnostik der Thorax- und parenchymatösen Oberbauch-Organe außerhalb der akademischen Zentren wenig geübt. Selbst die MR-Diagnostik der Leber, der die so genannten schnellen Sequenzen und die neuen Kontrastmittel den Status des Referenzverfahrens verschafft haben, ist nur an wenigen Stellen fest etabliert. Ähnlich zurückhaltend ist bisher die Nachfrage nach der MR-tomographischen Lymphknotendiagnostik. Wie kritisch die Kliniker aus dem Angebot der MRT wählen, zeigt eindrucksvoll das Beispiel der Gynäkologie. Während die MR-Mammographie voll anerkannt ist und in vielen onkologischen Problemfällen zu Rate gezogen wird, ist die MRT der Beckenorgane selbst vor großen Tumoroperationen die Ausnahme. Die besondere Bedeutung der MRT für die pädiatrische Onkoradiologie ist unschwer einzusehen. Freilich erfordern die Untersuchungen gerade bei Säuglingen und Kindern vielfach einen weit überdurchschnittlich hohen Zeitaufwand und das besondere Engagement des Personals.

Die MR-Angiographie, die man heute außer bei intrakraniellen Läsionen überwiegend nach Applikation eines paramagnetischen Kontrastmittels durchführt, wird von der Onkoradiologie kaum beansprucht. Die Gründe für die zurückhaltende Nutzung des Verfahrens sind identisch mit denen für den Rückgang der konventionellen Tumorangiographie. Lediglich die MR-Angiographie der Eingeweide hat sich manchenorts etablieren können. Die so genannte funktionelle MRT des Gehirns, die ihre Aussagen aus den aktivierungsabhängigen $\mathrm{pO}_{2}$-Änderungen des Blutes gewinnt, hält, obgleich sie erst seit etwa 10 Jahren betrieben wird, gerade Einzug in die Chirurgie der Hirntumoren. Der MR-Spektroskopie ist der Sprung in die klinische Praxis dagegen bisher nicht gelungen, und dies obwohl sie länger betrieben wird als die MR-Bildgebung und ihre Domäne der Tumorstoffwechsel ist. So faszinierend die Möglichkeit ist, mit ihrer Hilfe die relativen Anteile z.B. wasserstoff- und phosphorhaltiger Verbindungen volumenselektiv innerhalb einer Geschwulst zu messen, so bescheiden ist der Beitrag der Methode zur Diagnose und Differenzialdiagnose der malignen Tumoren geblieben. Eine Ausnahme von dieser Regel wie z.B. die spektroskopische Möglichkeit, Radionekrosen und Abszesse von Rezidiven intrazerebraler Tumoren einigermaßen zuverlässig abzugrenzen, bestätigt nur den wenig beflügelnden Gesamteindruck.

Die konventionelle nuklearmedizinische Tumordiagnostik hat im letzten Viertel des 20. Jahrhunderts Einbußen hinnehmen müssen. Von den angestammten Revieren sind nur die Schild- 
drüsen- und die Skelettdiagnostik nahezu unverändert erhalten geblieben. Für die parenchymatösen Organe, insbesondere die Leber, werden hingegen kaum noch morphologisch orientierte nuklearmedizinische Untersuchungen angefordert. Auch die ursprünglich großen Hoffnungen, die man auf die Antikörper-Szintigraphie von Tumoren gesetzt hat, haben sich nicht erfüllt. Dagegen haben die Rezeptor- und die «Sentinel node»-Szintigraphie zwei Segmente der diagnostischen Onkoradiologie besetzen können, die anderen nichtinvasiven Verfahren kaum zugänglich sind. Die Zukunft der Positronenemissionstomographie (PET) in der Onkoradiologie hat gewiss begonnen, ihre Gegenwart ist aber nicht so strahlend, wie man noch vor ein paar Jahren hoffte. Das Verfahren leistet in Teilbereichen hervorragende Dienste, sowohl in der Primärwie auch in der Rezidivdiagnostik maligner Tumoren. Ein bereits klassisches Beispiel ist der pulmonale Rundherd. Ein anderes weniger bekanntes ist das Monitoring und gegebenenfalls die Dosisanpassung der Chemotherapie maligner Lymphome durch sequenzielle PET. Das Spektrum neuer onkologischer Indikationen wächst aber nur langsam.

Um die Aussagen der von den verschiedenen Verfahren gelieferten morphologischen und funktionellen Daten über die Addition der Einzelinformationen hinaus zu verbinden und so das diagnostische Potenzial zu steigern, setzt man die «image fusion» (Bilddatenüberlagerung) ein. Dabei entstehen eindrucksvolle, in der Mehrzahl kolorierte zwei- und dreidimensionale Bilder (z.B. aus MRT und PET). Am meisten hat davon bisher die Strahlentherapie der Hirntumoren profitiert. Aber auch für andere therapeutische Interventionen und andere Organe wird diese aufwendige Technik von onkoradiologischen Forschergruppen getestet.

\section{Wettbewerb und Grenzerfahrungen}

So glänzend die Position der Radiologie unter den für die Onkologie tätigen diagnostischen Fächern ist, so wenig darf man ihre Defizite verkennen. Die Gründe für die punktuelle Unterlegenheit bzw. Unzuverlässigkeit der bildgebenden Diagnostik von Tumoren sind allerdings sehr verschieden.

Zum einen können manche Verfahren die Erwartungen, die durch die wissenschaftlichen Studien geweckt wurden, nicht erfüllen, wenn sie in voller klinischer Breite eingesetzt werden. $\mathrm{Zu}$ den enttäuschenden Resultaten tragen Mängel der apparativen Ausstattung und die fehlende Routine mancher Untersucher nicht wenig bei. Die Hauptursache ist jedoch anderswo zu suchen. Die klinischen Studien werden an einer ausgesuchten, intensiv kontrollierten Klientel mit hoher Erkrankungswahrscheinlichkeit durchgeführt. Die dabei ermittelten Werte für Sensitivität und Spezifität sind deshalb aus bevölkerungsmedizinischer Sicht zu hoch. Für den zu erwartenden diagnostischen Gewinn an unausgewählten Personen viel aussagekräftiger sind der positive und der negative Vorhersagewert. Zur Ermittlung der beiden Parameter, so fordert das Bayes'sche Theorem, ist aber die Kenntnis der Prävalenz unerlässlich. Je geringer sie ist, um so höher sind die Anforderungen an die Leistungsfähigkeit einer Methode. Bevor ein neues bildgebendes Verfahren z.B. für das Tumorscreening empfohlen werden kann, muss es diese epidemiologisch-statistische Hürde genommen haben.

Der allgemein anerkannte Erfolg der radiologischen Früherkennung von Mamma- und Bronchuskarzinomen darf nicht darüber hinwegtäuschen, dass der Einsatz bildgebender Verfahren zur Diagnose von Tumoren anderer Organe in frühen, unter Umständen kurablen Stadien enttäuscht hat. Die Hoffnungen, die man dabei noch in den 1980er Jahren auf die CT des Pankreas setzte, haben sich nicht erfüllt. Gerade die kleinen Bauchspeicheldrüsentumoren werden heute durch die Endosonographie zuverlässiger nachgewiesen als durch die CT. Allerdings ist auch das endoskopische Verfahren nicht zu einer Vorsorgemethode aufgestiegen. Ähnlich wie am Pankreas leisten die Schnittbildverfahren auch an der Prostata nur begrenzte Dienste für den frühen Nachweis von Karzinomen. Selbst die kontrastverstärkte MRT mit organadaptierten Spulen scheint dieser Herausforderung nicht gewachsen. So ist es auch nicht erstaunlich, dass Neuentwicklungen der bildgebenden Diagnostik für die präventive Onkologie wie etwa die CToder MR-Kolonographie (ergänzt durch die virtuelle Endoskopie) mit Skepsis betrachtet werden. Die Endoskopie ist als Referenzstandard der Darmkrebsdiagnostik (und minimal invasiven Therapie präkanzeröser Läsionen) zur Zeit und bis auf weiteres nicht ins Wanken zu bringen. Die vergleichenden Untersuchungen sind allerdings noch in vollem Gange. Immerhin kann man in der Bevölkerung Aufgeschlossenheit für die neue Variante der Darmspiegelung feststellen und damit eine hohe Compliance für die virtuelle Endoskopie auch im Wiederholungsfall prognostizieren.

Nach wie vor unbefriedigend sind die Ergebnisse der radiologischen Diagnostik während und nach der Behandlung maligner Tumoren. Dieses kritische Urteil gilt sowohl für die frühwie die spätposttherapeutische Phase und sowohl für die operative wie für die konservative Therapie. Die Radiologie wäre schlecht beraten, würde sie sich damit zufrieden geben, lediglich die klinisch manifesten Komplikationen der Behandlung sowie Lokalrezidive und metachrone Lymphknoten- und Fernmetastasen abzubilden. Doch das ehrgeizige Bemühen um lückenlose Dokumentation der onkologischen Therapie stößt rasch an methodische Grenzen. Das in den frühen Behandlungsphasen nahezu obligatorische fokale und perifokale Ödem maskiert mögliche Tumorresiduen und -ausläufer in die Umgebung. In den späten Phasen einer Behandlung erschweren Narben und die perifokale Fibrose die Erkennung und Größenbestimmung von Rezidiven. Besonders schwer wiegt dieser Nachteil z.B. bei der Kontrolle von Patienten, die wegen eines malignen HNO-Tumors operiert und/oder bestrahlt und/oder chemotherapiert wurden. Auch die Aussagekraft der MR-Mammographie bei der Rezidivsuche leidet darunter. Diese Schwierigkeiten wird die diagnostische Onko- 
radiologie wahrscheinlich erst dann überwunden haben, wenn es gelungen ist, neue organ- bzw. gewebespezifische Kontrastmittel für CT und MRT zu synthetisieren.

\section{Diagnostische und therapeutische Interventionen}

Der Fortbestand der Radiologie als einer eigenständigen Disziplin wäre heute gefährdet, würden ihre Vertreter nicht die aus der Analyse der Bilddokumente abgeleiteten Erkenntnisse durch diagnostische Interventionen vertiefen und außerdem konkurrenzfähige therapeutische Maßnahmen anbieten. In der Angioradiologie ist die Doppelfunktion des Diagnostikers besonders augenfällig. Aber auch die Onkoradiologie hat an dieser Entwicklung in vollem Umfang teilgenommen. Einige Verfahren sind sogar zu ausgesprochenen Prestigeobjekten geworden.

Statistik und Erfahrung lehren, dass unter den diagnostischen Interventionen die CT-gezielte Tumorbiopsie am weitesten verbreitet ist. Dieses Verfahren wird inzwischen in den meisten Krankenhausabteilungen und auch in vielen Praxen sicher beherrscht. Die Eingriffe verteilen sich auf zwei ungleich große Gruppen. Der ersten gehören diejenigen Läsionen an, bei denen ein oder mehrere Versuche, unter sonographischer Führung für die histologische Untersuchung geeignetes Material zu gewinnen, gescheitert sind. Ein typisches Beispiel dafür sind retroperitoneale Lymphome. In der zweiten, kleineren Gruppe sind die Läsionen versammelt, für die eine US-gezielte Punktion grundsätzlich nicht in Frage kommt. Dazu zählen vor allem Herde in der Lunge und im Skelett. Die Kosteneffektivität der CT-gezielten Biopsie ist in etlichen Untersuchungen nachgewiesen worden. Dabei konnte insbesondere demonstriert werden, dass das auf diese Weise erzielte histopathologische Resultat von den Klinikern mit großem Vertrauen angenommen wird und interdisziplinäre Therapiekonzepte anstößt. Der Ersatz der CT durch die MRT als Führungshilfe während der Biopsie ist nicht so rasch vorangekommen, wie man noch vor 5-10 Jahren hoffte. Mit der zunehmenden Verbreitung so genannter offener und halboffener MR-Geräte, die guten Zugang zum Patienten gewähren, wird sich diese Entwicklung jedoch beschleunigen.

Am weitesten fortgeschritten ist die klinische Prüfung der MR-gezielten Interventionen in der Senologie. Spezialisierte Zentren setzen die MR-geführten Eingriffe an der Mamma bereits in beträchtlichem Umfang ein. Der weiteren Verbreitung steht derzeit vor allem der Mangel an dedizierten Geräten entgegen. So wie heute röntgenmammographisch suspekte, nichtpalpable Herde präoperativ (z.B. mit Hilfe eines stereotaktischen Zielgeräts) markiert werden, wird man künftig auch mit den verdächtigen Befunden im MR-Mammogramm verfahren. Nur dann macht der breite Einsatz der hochsensitiven Methode überhaupt Sinn. Der Radiologe wird dabei in ausgewählten Fällen die Möglichkeit erhalten, durch den Einsatz der Stanzbiopsie den Brustkrebs minimal invasiv zu the- rapieren. Die enge Abstimmung mit dem Gynäkologen und Radiotherapeuten sowie die strenge Kontrolle durch den $\mathrm{Pa}-$ thologen sind unbedingte Voraussetzung für den langfristigen Erfolg dieses Konzepts.

Die Implantation von Stents zur palliativen Behandlung tumorbedingter Stenosen (z.B. der Gallenwege, der Speiseröhre oder des Tracheobronchialbaums) liegt heute fast ausschließlich in den Händen der Kliniker. Den Radiologen, die einen Teil der Entwicklungsarbeit geleistet haben, ist nur die Assistenzfunktion geblieben. Eine Ausnahme von dieser Verlagerung der Kompetenz bildet die Insertion großlumiger Gefäßstützen in die obere und untere Hohlvene bei tumorbedingter Einflussstauung. Da es sich meist um Patienten mit einem weit fortgeschrittenen Tumorleiden und lebensbedrohlicher Symptomatik handelt, ist der interventionelle Onkoradiologe zu notfallmäßigem Handeln aufgerufen. Der korrekt platzierte und entfaltete Stent beseitigt die akute Gefahr prompt; der Effekt der alternativ möglichen perkutanen Strahlentherapie setzt dagegen erst Stunden später ein.

Die präoperative Embolisation von Tumoren bzw. tumortragender Organe ist seit vielen Jahren bekannt, wird aber in ihrer ursprünglichen Form kaum noch gepflegt. Nur für Meningeome und gefäßreiche Nierenzellkarzinome fordert man diese Intervention noch gelegentlich an. Die vergleichenden Studien haben für die Kombination aus Embolisation und nachfolgender Resektion keinen eindeutigen kurz- oder langfristigen Behandlungsvorteil nachweisen können. Dagegen hat sich die Verbindung von Embolisation und lokaler hochdosierter Chemotherapie bei ausgewählten Tumoren der Mamma, des inneren Genitals und der Leber vor allem als (neo)adjuvante Maßnahme etabliert. Beim nichtoperablen hepatozellulären Karzinom gilt sie mittlerweile als Methode der Wahl. Anders als bei der üblichen lokalen Chemotherapie (via passageren oder permanenten Katheter) wird die Durchblutung der malignen Läsion durch ein flüssiges Embolisationsmittel gedrosselt und die Dauer des Kontakts zwischen Zytostatika und Tumor entsprechend verlängert. Die Chemoembolisation wird im Allgemeinen gut toleriert und kann einmal oder mehrfach wiederholt werden. Die Therapie von malignen Tumoren anderer Organe (z.B. der Lungen) auf diesem Wege steht am Forschungshorizont.

Während transarterielle Chemoembolisationen fast ausschließlich von interventionellen Onkoradiologen durchgeführt werden, erheben auf eine verwandte Therapieoption, die perkutane Ablation von Tumoren unter Einsatz von Radiofrequenz-, Laser- oder Kryosonden, andere Disziplinen einen Mitanspruch. Auch der Archetypus dieser Behandlungskategorie, die heute zumindest im europäischen Raum kaum noch übliche Instillation von Äthanol in Tumoren der Leber, wurde sowohl von Radiologen als auch von Vertretern der klinischen Fächer geübt. Das Zielorgan und die Zielläsionen, in der Regel Metastasen, haben sich freilich nicht geändert. Die Grenzlinien des fachspezifischen Einsatzes der neuen Verfahren sind inzwischen ebenfalls recht klar geworden. Wenn der 
Herd sonographisch zuverlässig geortet werden kann, erscheint die lokale Zerstörung des Tumors unter der ausschließlichen Kontrolle des ubiquitär verfügbaren Ultraschalls möglich. Wenn der Chirurg einen oder mehrere Herde nicht resezieren, aber eindeutig lokalisieren kann, wird die Ablation vorzugsweise intraoperativ durchgeführt. In den meisten anderen Fällen ist aber der Radiologe zu derartigen Interventionen aufgerufen, weil er mit CT und MRT über hervorragende Steuerungsinstrumente verfügt. Die MRT ermöglicht zusätzlich die unmittelbare Beobachtung der Tumordestruktion. Stets wird der Therapieerfolg im weiteren Verlauf computer- und/oder kernspintomographisch kontrolliert; dabei spielen Ausmaß, Verteilung und Geschwindigkeit der Akkumulation von Kontrastmitteln in den Herden eine fundamentale heuristische Rolle. Nach der derzeitigen Datenlage verlängert die perkutane Ablation das Überleben von Tumorpatienten mit Lebermetastasen beträchtlich. Gegen die Studien ist jedoch mit Recht der Einwand erhoben worden, dass zum Vergleich historische Kollektive herangezogen und die Komorbidität sowie andere Todesursachen nicht ausreichend berücksichtigt worden sind. Außerhalb kontrollierter Studien wird die Effektivität der perkutanen Verfahren nahezu regelhaft durch die Wirkungen konkomitierender Behandlungsversuche überdeckt werden.

\section{Intelligente Konzepte}

Wie dieses und andere Beispiele zeigen, ist der Onkologe in der Praxis gut beraten, wenn er die Studien aus der diagnostischen und interventionellen Tumorradiologie mit ebensoviel Respekt wie Zurückhaltung rezipiert. Mit Respekt, weil die Durchführung derartiger Untersuchungen großen Organisationsaufwand erfordert, mit Zurückhaltung, weil die Resultate allzu oft vorschnell auf die allgemeine Klientel extrapoliert werden. Das Konzept der evidenzbasierten Medizin wird auch in der Onkoradiologie nur dann akzeptiert werden, wenn die Studienresultate sine ira et studio dem Härtetest der bevölkerungsmedizinischen Erprobung unterworfen werden. Nur die Verfahren, die sich in beiden Prüfungen bewährt haben, können dauerhaft praxisfest sein.

Der diagnostische Radiologe ist stolz darauf, die früher übliche so genannte Stufendiagnostik grundsätzlich verlassen zu haben und auf eine gezielte Fragestellung mit der Empfehlung für das im Allgemeinen treffsicherste Verfahren zu reagieren. Die umfassenden Kenntnisse und Erfahrungen bei der Anwendung der bildgebenden Verfahren legitimieren seine Vor- schläge. In der Onkoradiologie sind allerdings noch gewisse Residuen aufwendiger sequenzieller Diagnostik zu beklagen. Ein gutes Beispiel dafür sind die fokalen Leberläsionen. Einmal entdeckt, werden sie nicht selten multimodal zu verifizieren und zu charakterisieren versucht, bis endlich die zielführende bildgebende Methode oder die Biopsie eingesetzt wird. Nur enger interdisziplinärer Kontakt und Verzicht auf Besitzstandsdenken kann derartige Testkaskaden künftig verhindern. Auf der anderen Seite bedeutet die Entscheidung für ein bestimmtes Verfahren nicht ohne weiteres, dass sämtliche damit möglichen Spielarten der Bildgebung, wie sie etwa für die MR-Diagnostik des Pankreas entwickelt worden sind, innerhalb einer Sitzung in Anspruch genommen werden. Ein derartiges Vorgehen spart zwar Zeit, generiert aber unter Umständen eine Reihe entbehrlicher Befunde.

\section{Moleküle im Bild}

Die sich anbahnende Kooperation von bildgebender Diagnostik und Molekularbiologie im Rahmen der Gentherapie wird auch und gerade die Onkoradiologie beflügeln. Übergeordnetes Ziel der auf MRT und PET fußenden neuen Techniken ist es, molekulare Prozesse, d.h. die Expression von Genen und die Funktion von Proteinen, abzubilden. Dazu bedarf es der Entwicklung so genannter Sonden, die eine Krankheit auf der Ebene der molekularen Pathologie erkennen lassen und sozusagen als molekülspezifische Kontrastsubstanzen angesehen werden können. Bevor diese Marker beim Menschen eingesetzt werden, muss ihre Verträglichkeit gesichert sein und eine Applikationsform zur Verfügung stehen, die garantiert, dass sie im Gewebe ausreichend akkumulieren und mit marktüblichen Geräten nachgewiesen werden können. Als besonders erfolgversprechend haben sich auf diesem Sektor die Versuche erwiesen, Genprodukte wie Melanin, die intrinsische paramagnetische Eigenschaften aufweisen, MR-tomographisch zu studieren.

Während in anderen Bereichen die interventionellen Leistungen der Radiologie den diagnostischen im Allgemeinen nachfolgen, ist es auf dem Sektor der Gentherapie umgekehrt. Die bildgesteuerte lokale Applikation von Therapievektoren ist vereinzelt bereits klinisch realisiert worden. Die Leistungsfähigkeit von CT und MRT als Führungsinstrumente zur Injektion genetischen Materials ist anerkannt. Bereits jetzt ist abzusehen, dass Katheter, Ballons und Gefäßstützen als Materialträger in die Gentherapie maligner Tumoren einziehen werden. 